\title{
Erklärung auf dem physiologischen Mechanismus von Inflammation, besonders bei Reparation
}

Von

Kineshiro NAGAI*

English Summary

The proliferation of inflammatory granulations can be considered as a functional expression of the mesenchyme in the presence of an inflammation. The author thinks a chemical agent promoting this mechanism to be a synenergic system of carnosine and corticoide.

For an effective vital defense, it requires not only corticoide alone, but there must be also some kind of protective response inducing substances (PRIS). Further, the author touches on an anti-infectious and anti-allergic action of carnosine.

\section{Einleitung}

Bei der chirurgischen bzw. pharmazeutischen Behandlung der Wunde handelt es sich um nur die Blutstillung, die Prophylaxe der Infektion, die Antiphlogose und die Schmerzstillung. Da es keine Behandlungmögslichkeit für die Beförderung der Wundeheilung gibt, muss man nur auf die Naturheilung warten. Es war als eine bisherige medizinische Behandlung das beste Verfahren, das man die innere und äussere Faktoren, die die natürliche Heilung verhindern, beseitigt und auf die natürliche Heilung zu warten. Wenn jedoch der physiologische Mechanismus für die Wiederherstellung selbst geschädigt ist, verspätet sich die Heilung oder ist sie sogar unmöglich. In diesem Fall ist es notwendig, den physiologischen Mechanismus für die Wiederherstellung zu aktivieren und die Heilung dadurch zu befördern.

Man sieht klinisch öfter, dass sich die Entzündung als eine Abwehrreaktion befindet, und trotzdem wegen der Schädigung des Wiederherstellungsmechanismus die Heilung sich vezögert. Es wäre unterschiedlich von der Wundheilung durch die antiinflammatorische Substanz, die Selye aufgestellt hat, eine enue Entwicklung in der Richtung der physiologischen Behandlung der Entzündung den Verlauf der Gewebeswiederherstellung durch Entzündung zu erklären.

\section{Betrachtungen des Abwehrvorgangs und des Inflammationsvorgangs nach der Entzündungstheorie}

Bei dem Studium der Entzündung liegt der folgende Gesichtspunkt im Grund,

\footnotetext{
* 永井甲子四郎: Institut für Physiologie, Nihon Universität, Tokyo
} 


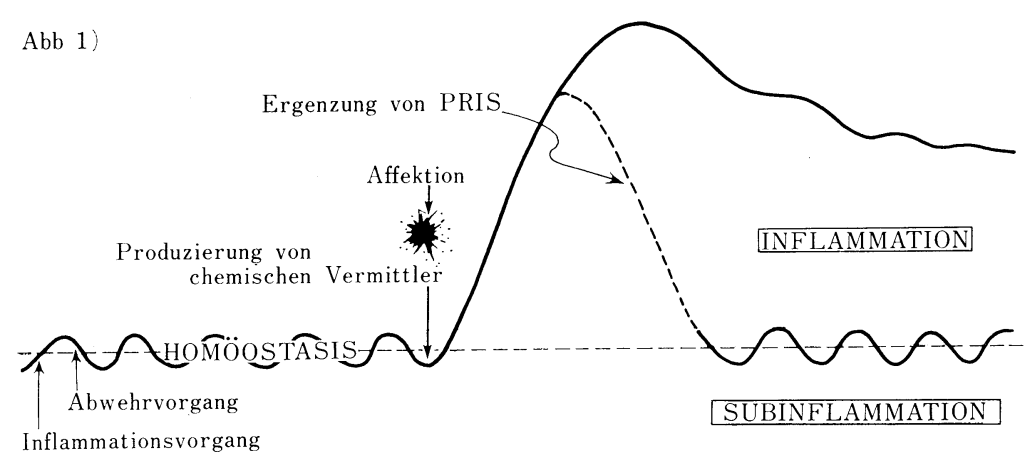

Abb. 1

Die pharmakologische Untersuchung über der Entzündung wurde an den Erscheinungen nach dem Entzündung verursachenden Reiz durchgeführt. Physiologisch wird der ganze Bereich einschliesslich der Subinflammation gegen Inflammation untersucht. Die Homöostase stellt die Wiederholung zweier Vorgänge dar. Wenn die Inflammation gleich nach ihrer Entstehung wieder repariert werden kann, gibt es kein Problem. Da der Inflammations-vorgang lange dauert, wird die Beschleunigung des Abwehrvorgangs erfordert.

dass sich die Ursache der Entzündung zufällig ereignet, und deswegen die Entzündung auch eine akzidentielle Reaktion des Gewebes ist. Die pharmakologische bzw. biochemische Forschung der Entzündung beschränkt sich deshalb darauf, die Gewebesreaktion an der Entzündung zu studieren, die an der Stelle angefangen ist, wo der Affektion für die Entstehung der Entzündung gegeben wurde. In dieser Richtung erforschte man pharmakologisch und pharmazeutisch ausschliesslich die Hemmung der Entzündung und die antiinflammatorischen Präparate wurden bisher entwickelt.

Die Entzündung entsteht jedoch in der Tat in der Folge der Zerstörung der Homöostase des Mesenchyms (Abb. 1). Das ist zwar eine Zerstörung der Homöostase, ist jedoch nicht ganz ausser der physiologischen Kontrolle. Das ist eine Aktivierung des Homöostasemechanismus, der selbstverständlich eine Vollbringung der Wiederherstellung voraussetzt.

Der Autor [1, 2] unterscheidet das Mechanismus der Homöostase in den Inflammationsvorgang und den Abwehrsvorgang, und stellte die folgende Hypothese über die Substanzen auf, die mit diesen Vorgängen in Bezieung stehen, um den Entzündungsmechanismus zu erklären (Abb. 2, 3).

Nämlich nennt der Autor vorläufig die Substanz, die zusammen mit Corticoid den Inflammationsvorgang induziert, IRIS (Inflammation Response Inducing Substances), und die Substanzen, die zusammen mit Corticoid den Abwehrvorgang induziert, PRIS (Protective Response Inducing Substances), und nimmt an, dass diese 4 Substanzen einander die Homöostase des Gewebes aufrechterhalten.

In dem Zustand der Homöostase wiederholen sich die zwei Vorgänge, der Inflammations- und der Abwehrvorgang, in einer einheitlichen Stärke und rhythmisch. Wenn das Gleichgewicht zwischen den beiden Vorgänge gebrochen wird, entsteht die Entzündung (Abb. 1, 4).

Das Gleichgewicht wird durch die abnormale Funktionssteigerung von IRISCorticoid gebrochen und die natürliche Heilung geschieht durch die Funktionssenkung 
Abb. 2

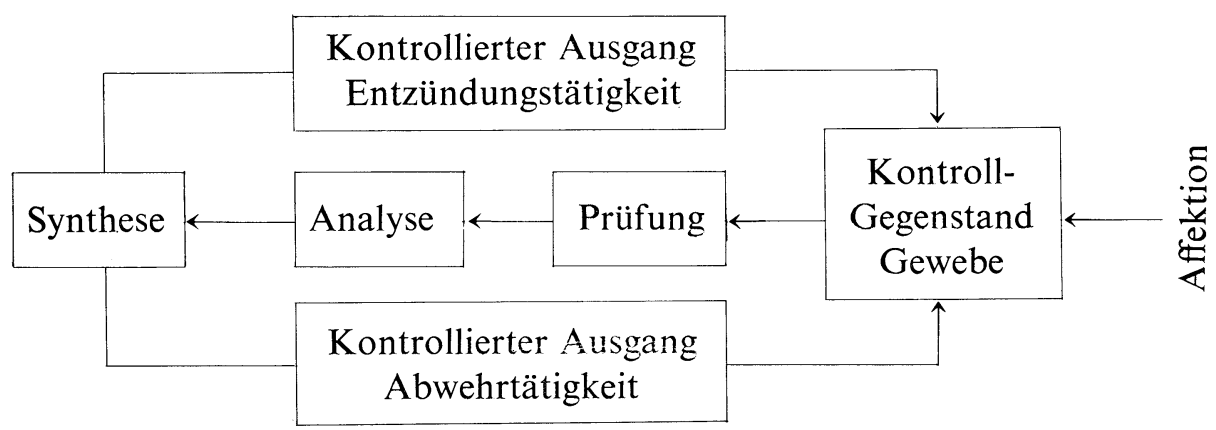

Abb. 3

Arbeitshypothese für die chemischeh Vorgänge bei der Entzündung.

(NAGAI 1967)

Corticoid + IRIS (Inflammatory response inducing substances)

Entzündungsvorgang

Homöostatische Gewebsfunktion

Abwehrvorgang

Abb. 2, 3

Die Homoostase des Gewebes wird durch zwei Vorgänge aufrechtgehalten. In Abb. 2. ist der Kontroll-mechanismus schematisch dargestellt. Abb. 3. zeigt die Hypothese von NAGAI, dass die zwei Vorgänge durch die Zusammenwirkung der Substanzen IRIS, PRIS und Corticoid reguliert werden. 


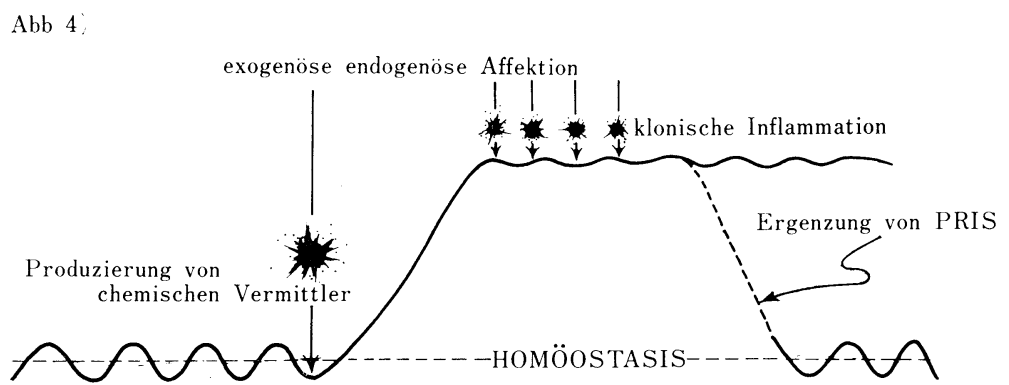

Abb. 4

Durch den klonischen Reiz und den schlecht zirkulierenden inneren Reiz wird die Entzündung klonisch. Da der Abwehrvorgang gehemmt ist, kommt die Reparation nicht zum Funktionieren. Wenn PRIS in einer Dosis gegeben wird, die den Abwehrvorgang gegen den äusseren und inneren Reiz gut erhalten kann, wird der Abwehrvorgang beschleunigt, und kommt der Inflammationsvorgang zum Abschluss, damit die Homäostase wiederhergestellt wird. Ein Beispiel dafür ist das gute Behandlungsergebnis von GABOB und GOBAB auf den Decubitus.

von IRIS-Corticoid oder die Funktionssteigerung von PRIS-Corticoid. Der Vorgang der natürlichen Heilung, d.h. der Abwehrvorgang, kann deswegen durch das Studium von PRIS-Corticoid als physiologische Wirkstoffe erklärt werden.

\section{Nachweis von PRIS, die Substanz, die den Abwehrvorgang induziert.}

Bei der experimentellen Entzündung in der Stelle, wo sich grösstenteils Mesenchym befindet, tritt der Abwehrvorgang im Gewebe in Reaktionen "demarkatio", "remotio" und "absorptio" auf. Im histologischen Gesichtspunkt handelt es sich dabei um "granulatio" und "absorptio", Wenn es durch die experimentelle Untersuchung nachgewiesen wurde, dass sich die diese Reaktionen beschleunigende Substanz im entzundlichen Gewebe befindet, und wenn es nach der Verabreichung der Substanz in etwa derselben Menge wie im Gewebe gelang, den Abwehrvorgang zu befördern, dann kann geschlossen werden, dass die Substanz ein physiologischer Wirkstoff ist, der mit dem Abwehrvorgang in Beziehung steht.

Bei der Untersuchung wird die antiödematose Wirkung gegen das Ödem, das durch Carrageenin bzw. Trauma oder durch andere Methode hervorgerufen wurde, als Parameter registriert. Auch mit der Granuloma-Methode wird die Untersuchung durchgeführt, die seit Selye bei der Prüfung der antiinflammation Wirkung gerne angewandt wird. Wir haben ein kleines Filterpapier, das mit der $7 \%$ igen Formalinlösung getränkt ist, s.c. in die scapularen Region von Ratten eingelegt und nach einer Woche herausgenommen, um die Menge der Granulation, die durch die Demarcatio-Reaktion entstanden ist, als ein Parameter zu registrieren.

\section{1) Wirkung von $G A B O B$ und $G O B A B$}

Die physiologischen Wirkungen von $\omega$-Aminosäuren unterscheiden sich durch die Anzahl des C-Atoms in seiner C-Kette. HAYASHI u.M. (3) untersuchten die Wirkungen verschiedener $\omega$-Aminosäuren auf das motorische Zentralnervensystem 
und fanden, dass 4-amino-3-hydroxy-Buttersäure (GABOB) die beste Hemmwirkung hat. HAYASHI schreibt (3), dass sich die Substanz auf die Hemmung des motorischen Zentral-nervensystems bezieht. Die Substanz wird klinisch als ein Epilepsie-Hemmstoff mit Erfolg verwandt.

NAGAI u.M. [4, 5, 6, 7] untersuchten die Antiödematösewirkung verschiedener $\omega$-Aminosäuren und fanden, dass GABOB und 4-hydroxy-3-amino-Buttersäure (GOBAB) antiödematös wirken. Sie schreiben auf Grund der charakteristischen Wirkung von GABOB bzw. GOBAB auf Entzündung, die im folgenden beschrieben wird, dass die Kombinations-verabreichung von Cortion-GABOB physiologisch mit dem abwehrmechanismus des Körpers in Beziehung steht.

Die pharmacologischen Wirkungen von GABOB bzw. GOBAB auf Entzündung:

(a) $200 \mathrm{mg} / \mathrm{kg}$ von Substanzen i.v. wirken gegen das Ödem antiödematös, das durch Carrageenin oder Trauma hervorgerufen wurden.

(b) Die Wirkung kommt bei den Nebennieren resektierten Tieren nicht zur Erscheinung. Die antiödematöse Wirkung kommt jedoch nach der Kombinationsverabreichung mit Cortison wieder zur Erscheinung.

(c) Auch bei normalen Tieren verstärkt sich nach der Kombinationsverabreichung mit Cortion bzw. ACTH die antiödematöse Wirkung. Nach der Kombinationsgabe mit DOCA ist die Wirkung nicht zu beobachten.

(d) Nach der Behandlung mit der Formalin-Filtrierpapier-Peletmethode wird im Vergleich mit dem Kontrolle die Granulierung beschleunigt.

(e) Nach der Kombinationsverabreichung mit Cortison wird die Granulierung im Vergleich mit der nach der alleinigen Verabreichung beschleunigt.

Zusammenfassend kann man sagen, dass GABOB bzw. GOBAB zusammen mit Cortison die Gewebesreaktionen "granulatio" und "absorptio" beschleunigen. Es ist auf Grund der klinischen Erfahrungen zu verstehen, anzunehmen, dass Cortison mit der Abwehr des Organismus in Beziehung steht. Exogenische Cortison hat jedoch eine Antiabwehrwirkung. Das Problem wurde von mehreren Forschern qualitativ und quantitativ untersucht, jedoch gibt es heute noch keine endgültige Erklärung.

GABOB bzw. GOBAB rufen zusammen mit Cortison eine Abwehrreaktion hervor, d.h. ist es anzunehmen, dass die Substanzen die Abwehrwirkung von Cortison induzieren. Es ist anzunehmen, dass Cortison durch das Zusammensein mit GABOB bzw. GOBAB im Gewebe eine Abwehrwirkung entfaltet. Es ist daraus zu vermuten, dass GABOB bzw. GOBAB der Substanz PRIS nach unserer Hypothese entsprechen. Kann GABOB dann der physiologische Wirkstoff PRIS sein Hier entstehen eiinge Schwierigkeiten. Obwohl sich sein Betain als Carnitin reichlich im Gewebe befindet ist das Dasein von GABOB noch nicht nachgewiesen. Die Large ist dieselbe auch bei GOBAB.

Auch die wirksame Dosis gegen Ödem. $200 \mathrm{mg} / \mathrm{kg}$, ist zu hoch, wenn auch vorausgesetzt wird, dass sich GABOB in der Konzentration ähnlich wie bei Carnitin befindet.

Die zwei oben genabbten Tatsachen sind die Gründe dafür, dass man GABOB bzw. GOBAB noch nicht als physiologische Wirkstoffe endültig feststellen kann. Der Autor nimmt an, dass GABOB bzw. GOBAB ein Model von PRIS sind. HAYASHI stellt auf, dass sich GABOB im Gehirngewebe befindet und als ein physiologischer 
Wirkstoff das motorische Zentralnervensystem hemmt. GABOB wirkt im Mesenchym und im Gehirngewebe anders. Es ist jedoch phyiologisch sehr interessant, dass die Substanz gegen Erregung des motorischen Zentralnervensystems und Entzündung im Gewebe abwehrend wirkt.

Wenn man im GABOB nicht ein einfaches Antispasmodica, sondern eine Substanz sieht, die mit der Homöostase des Zentralnervensystems in Beziehung steht, ist es dann zu verstehen, dass GABOB klinisch nicht als ein Antispasmodica, sondern für die Stabilisierung der Funktion des Zentralnervensystems verwandt wird. Homocarnosin, das eine ähnliche Wirkung wie Carnosin hat, befindet sich im Gehirngewebe. M. KOSAKA u.a. [8, 9] behandelt Epilepsie mit dieser Substanz. MORI u.M. (10) stellen auf, dass die Substanffi das "limbic" System des Zentralnervensystems hemmt. Der Autor nimmt nach Analogie von der Wirkung auf Entzündung an, dass Carnosin bzw. Homocarnosin mit der Homöostase der Funktion des Gehirns in Beziehung steht.

\section{2) Wirkung von Carnosin bzw. Homocarnosin ${ }^{[11,12]}$}

Carnosin wurde 1900 im Fleischextrakt und Homocarnosin 1961 im Findengehirn gefunden. Seitdem wurden die Substanzen physiologisch, pharmakologisch und biochemisch viel studiert, trotzdem ist die physiologische Wirkung dieser Peptiden noch nicht endgültig erklärt. Die wirksame Dosis von Carnosin bzw. Homocarnosin gegen das Carrageenin-Ödem bzw. traumatisches Ödem beträgt ca. $30 \mathrm{mg} / \mathrm{kg}$ i.v. Die antiödematöse Wirkung der Substanzen kommt bei den Nebennieren resektierten nicht zur Erscheinung, aber sie kommt wieder, wenn die Substanzen zusammen mit Cortison verabreicht wird. DOCA wirkt dabei nicht wie Cortison.

Auch bei Granulation wirken diese Substanzen ähnlich wie GABOB, d.h. zusammen mit Cortison beschleunigen sie Granulierung. Die wirksame Dosis von Carnosin, ca. $30 \mathrm{mg} / \mathrm{kg}$ i.v., entspricht vermutlich ca. $1 / 40$ der Konzentration im Skeletmuskel. Die Menge bedeutet jedoch eine pharmakologisch wirksame Menge gegen das experimentelle Ödem. Es ist anzunehmen, dass die Substanz physiologisch in einer kleineren Menge wirksam ist. Die Tatsache, dass Carnosin in einer viel kelineren Menge als die Konzentration im Gewebe zur Wirkung kommt, weist hin, dass die Substanz als eine Art PRIS physiologisch wirken kann. Wie schon erwähnt wurde, befindet sich Homocarnosin im Gehirn und nimmt an die Funktion des Zentralnervensystems teil.

\section{Antiinfektiose Wirkung von Carnosin bzw. Homocarnosin}

Die Untersuchungsgruppe vom Institut Devi Tomas (Cincinati) wies schon nach, dass Carnosin, besonders Homocarnosin gegen Staphylococcen-Infektion abwehrend wirken [13, 14, 15]. Ca. $213 \mathrm{mg} / \mathrm{kg}$ dieser Substanzen bei Maus wirken antiinfektiös und senken die Mortalität durch Infektion herab. Die wirksame Menge der Substanzen in der Stufe der Gewebeschomöostase, wo die Infektion entsteht oder gehemmt wird, ist vermutlich sehr klein. Deswegen ist die physiologische Bedeutung dieser Substanzen für die Hemmung der Infektion gross. In Abb. 5 ist der Zusammenhang zwischen der Infektionsentstehung und dem Abwehrvorgang dargestellt. Durch die Gabe von PRIS 
wird der Abwehrvorgang verstärk und die Infektionsentstehung gehemmt. Carnosin bzw. Homocarnosin wirken gegen den chemischen Vermittler, der durch Invasion der Bakterien entstanden, und halten die Homöostase des Gewebes aufrecht.

Abb 5 ;

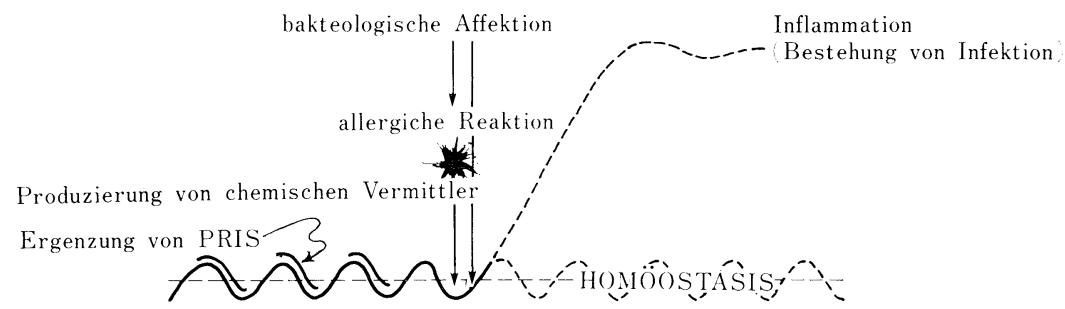

Abb. 5

Eine bakteriologische Affektion wirkt als ein chemischer, mechanischer und allergischer Reiz und lässt chemische Vermittler entstehen, um den Mechanismus der Homäostase zu beschädigen und Entzündung-Infektion hervorzurufen. Wenn dabei PRIS verabreicht wird, kommen die Reparation beschleunigende Wirkung und die antagonistische Wirkung gegen Bradykinin zur Erscheinung und wird infolgedessen die Infektion prophylaktisch gehemmt.

\section{Antiallergische und antibradykinin Wirkung von Carnosin [6]}

Carnosin hemmt das Arthus-Phänomen (Tab. 1) und die lokale Anaphylaxie des Darmtraktes von sensibilisierten Meerschweinchen. Die Hemmkonzentration beträgt 10I/...5 10I/ mol (Abb. 6(. 10I/ mol Carnosin bzw. Homocarnosin wirken antagonistisch gegen Kontraktion des Darmtraktes von Meerschweinchen durch Bradyktinin (Abb. 7, 8(. Beim Anaphylaxieschock von Meerschweinchen wirken die Substanzen prophylaktisch oder verschiebt den Tod der Tieren bedeutend (Tab. 2).

Der Autor nimmt an, dass die Hemmwirkung der Substanzen gegen die allergische Reaktion auf die Antibradykininwirkung beruht. Bei der Hemmwirkung gegen die Arthusreaktion beruht jedoch meistens auf die Reparationsbeschleunigung.

Auch die Abwehrwirkung gegen Infektion beruht auf

1. die Hemmung der allergischen Reaktion und

2. die Reparationsbeschleunigung.

Durch die oben genannten beiden Wirkungen wird die Gewebeshomöostase gegen Infektion aufrechtgehalten. Bei der Infektionsentstehung hat die allergische Reaktion eine grosse Bedeutung. Die oben erwähnte Hemmwirkung gegen das Arthus-Phänomen ist von Bedeutung, um die antiinfektiöse Wirkung der Substanzen zu verstehen. 
Tab. 1

Der inhibitorische Effekt von Carnosin auf das Arthus-Phänomen (Die Tiere wurden mit dem Kaninchenserum enthaltenden Pferdeserum Antikorper sensibilisiert. Die geschriebene Dosis wurde 6 mal jede zweite Stunden und 4 mal jede vierte Stunden gegeben. Die Haut wurde in 48 Stunden.

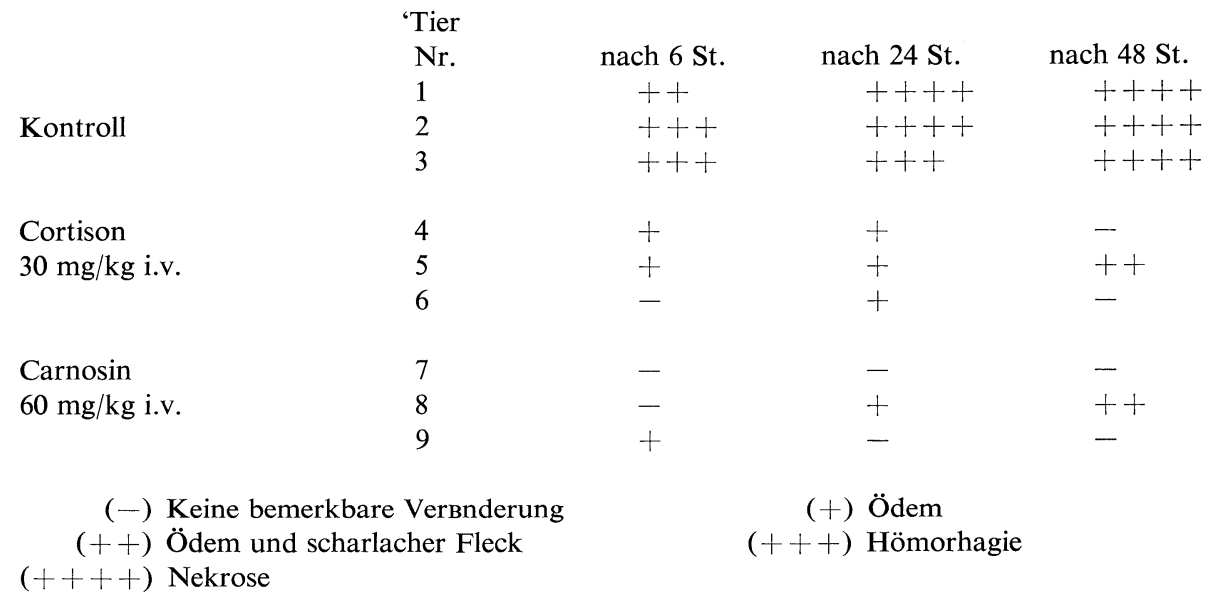

Tab. 2

Effekt von Carnosin beim Blutserumschock, der durch 0.5 mol Pferdeserum verursachtet wurde (10 männliche Meerschweinchen 250-350 g)

\begin{tabular}{|c|c|c|c|}
\hline $\begin{array}{l}\text { Tier } \\
\text { Nr. }\end{array}$ & Dosis & $\begin{array}{l}\text { Zeitdauer bis } \\
\text { zum Tod }\end{array}$ & $\begin{array}{c}\text { Mortalität } \\
(\%)\end{array}$ \\
\hline 10 & Kontroll & 3 min 47 sek & 100 \\
\hline 10 & Carnosin $100 \mathrm{mg} / \mathrm{kg}$ i.v. & 14 & 60 \\
\hline 10 & Carnosin $200 \mathrm{mg} / \mathrm{kg}$ i.v. & 54 & 80 \\
\hline
\end{tabular}

Abb. 6

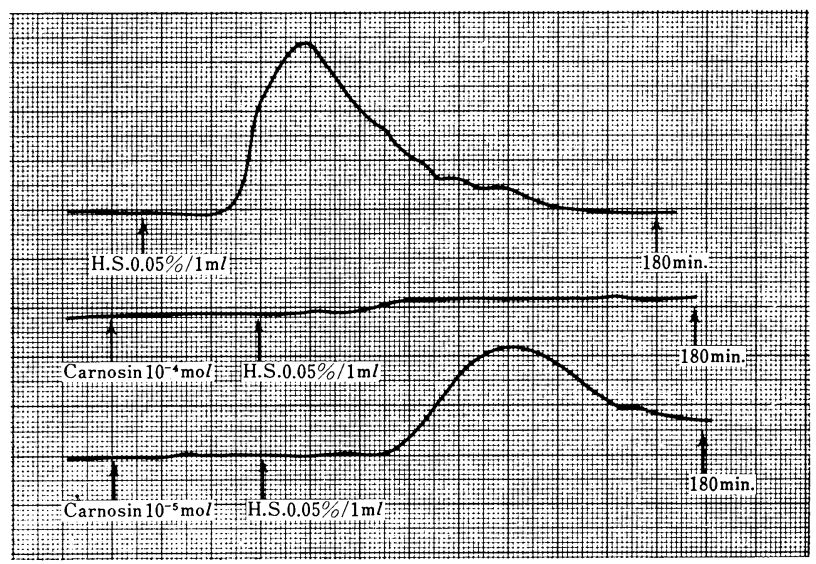

Der inhibitorische Effekt von Carnosin gagen lokale anaphylaktische Erscheinung (sensibilisierter Darmtrakt von Meerschweinchen) 
Abb. 7

Der Antibradykinin-Effekt von Carnosin (Darmtrakt von Meerschweinchen)
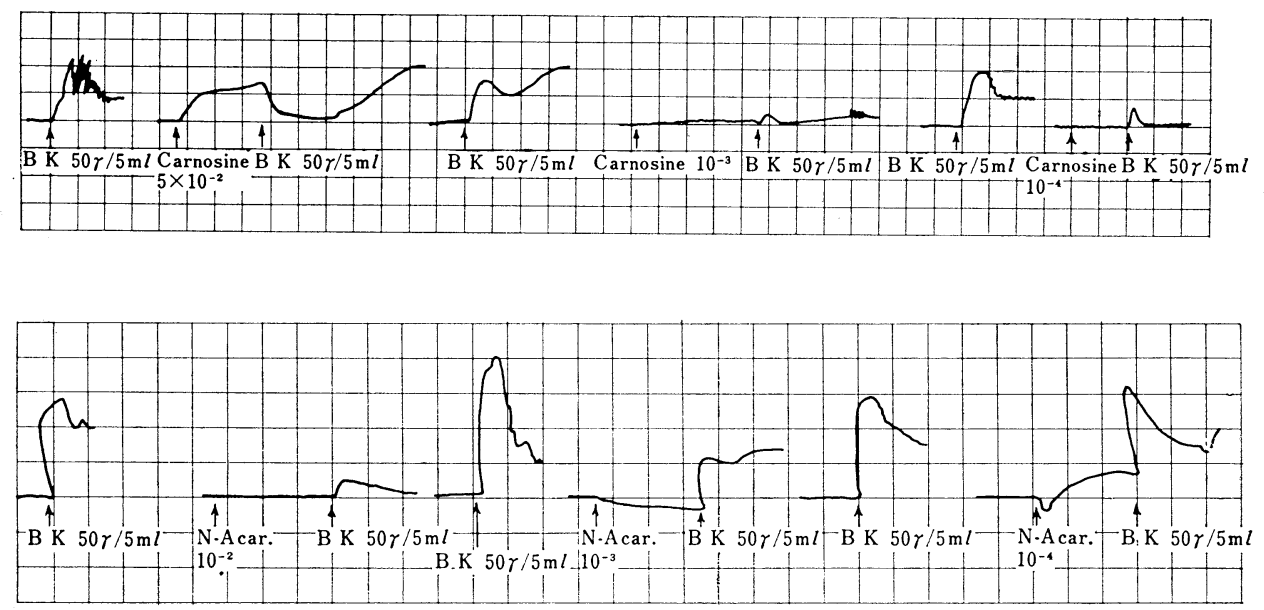

Abb. 8

Der Antibradykinin-Effekt von Homocarno(in (Darmtrakt von Meerschweinchen)
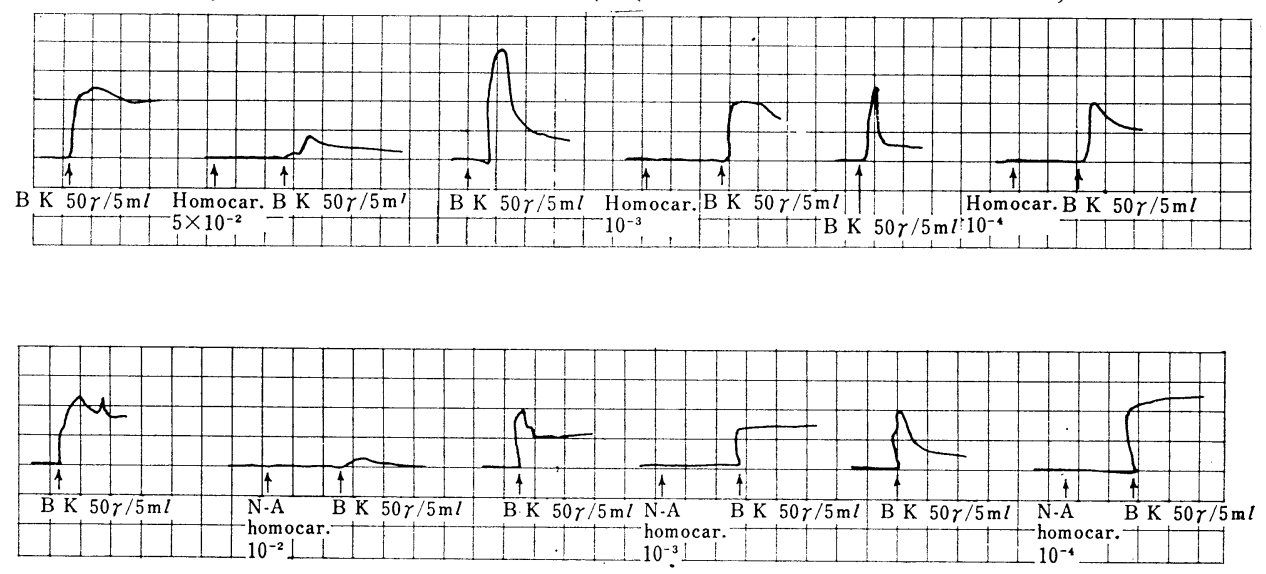

\section{Die zweite Hypothese von Nagai}

Der Autor hat die Substanz PRIS nachgewiesen, die den Abwehrwirkung induziert.

Über IRIS, das den Entzünhungsvorgang induziert, Corticoide, die an beide Vorgänge teilnehmen, und besonders Corticoid, das an den Entzündungsvorgang teilnimmt, gibt es noch keine endgültige Erklärung. 
Die Homöostase des Gewebes wird durch rhythmische Wiederholung der zwei Vorgänge aufrechtgehalten.

Wie hängen dann PRIS und IRIS, die je einen Vorgang induzieren, einander zusammen? Der Autor nimmt an, dass es auf das Verhältnis PRIS/IROS ankommt, d.h. wenn sich IRIS mehr als PRIS befindet, wird die Entzündung induziert, und wenn sich PRIS mehr als IRIS befindet, wird der Abwehrvorgang induziert.

Bei beiden Vorgängen stellen IRIS und PRIS periphere Bedingungen im Gewebe dar. Wie ist es also bei Corticoide, die zentrale Bedingungen in Nebennieren darstellen? Beim Abwehrvorgang spielt Cortison eine Hauptrolle, beim Entzündungsvorgang ist es noch nicht geklärt.

Selye erklärt in seiner Theorie über den General-Adaptationssyndrom, dass das Verhältnis DOCA/Cortison die Homöostase des Gewebes entscheidet. Dabei soll DOCA prophlogistisch und Cortison anti-phlogistisch wirkt. Er stellt auf, dass 2 Vorgänge im Gewebe durch das Verhältnis DOCA/Cortison zur Erscheinung kommen. Der Verfasser vermut, dass DOCA/Cortison mittles IPIS/PRIS den Abwehrvorgang und Inflammationsvorgang induzcert, und dadurch die Homöostase des Gewebes aufrechtgehalten wird.

\section{Literatur}

[1] K. Nagai, Eine Arbeithypothese über die physiologie der Entzündung und die Substanzen, die den Entzündungs- und Abwehrvorgang auslösen; J. Nihon Univ. Sch. Dent., Vol. 11, No. 1, p. 16-22, (1969).

[2] K. Nagai et al: Antiinflammatory agents and physiological concepts of their screening; J. Nihon Univ. Sch. Dent., Vol. 10, No. 1, (1968).

[3] T. Hayashi: Neurochemistry of convulsion; Dainihon-Tosho Co., Ltd. Tokyo, (1959).

[4] K. Nagai et al: Antiinflammatorische Wirkung der 4-Hydroxy 3-Amino Buttersüure; J. Nihon Univ. Sch. Dent., Vol. 9, No. 1, p. 35-42, (1967).

[5] M. Katagiri: Antiinflammatory action of various $\omega$-Amino acid; Nihon Univ. Dent. J. (in pub.)

[6] K. Nagai et al: Studies on the synergistic action and anti-ulcerous activity of cortisonGABOB; Arzneim.-Forsch. (Drug Res.), Vol. 21, s. 96-97, (1971).

[7] M. Sekiguchi: Studies on the antiinflammatory effect of 4-hydroxy 3-aminobutyric acid (GABOB); Nihon Univ. Dent. J., Vol. 44, 560-567, (1970).

[8] M. Kosaka: Jap. J. Brain Physiol. No. 40, p. 84-95, (1963).

[9] S. Takagi and M. Araki: Jap. J. Brain Physiol. No. 61, p. 138-142, (1965).

[10] A. Mori et al: Jap. J. Brain Physiol. Vol. 34, 0. 40-43, (1963).

[11] K. Nagai: Physiological implication of Carnosine on the inflammation-with reference to its inhibitory action of allergy and the vital defence mechanism-J. Nihon Univ. Sch. dent. Vol. 13. No. 1 p. 1-11 (1971)

[12] K. Nagai et al.: Die physiologische Bedeutung von Carnosin und Homocarnosin bei der Inflammation; J. Nihon Univ. Sch. Dent. Vol. 13, No. 1, 0.1-12, (1971).

[13] K. Tanaka, et al.: Activity of Homocarnosine and other compounds against Staphyloccal infection in mice, Appl. Microbiol., Vol. 19, p. 813-817, (1950).

[14] A. J. Mukkada et al.: Prophylactic effect of Homocarnosine on experimental Staphylococcal infection in mice, Appl. Microbiol., Vol. 16, p. 641-645, (1969).

[15] Y. Tsuchiya et al.: Effect of -amino acid and related compounds on Staphylococcal infection in mice; a combined prophylactic-therapeutic procedure, Appl. Microbiol., Vol. 19, p. 813-817, (1970). 
[16] K. Nagai u.s.w.: Die hemmende Wirkung von Carnosin auf verschiedene allergische Reaktionen; Arzneim.-Forsch. (Drug Res.) Vol. 21, s. 1222-1225, (1971).

[17] K. Nagai und H. Sugiyama: Klinische Wirksamkeit von 4-Hydroxy-3 Amino-Buttersäure (GOBAB) bei Dekubitus Erklärung eines neueren Begriffes von Antiinflammation; J. Nihon Univ. Sch. Dent., Vol. 14, No. 2,(1972). 